\title{
Quality of Life after Coronary Revascularization in Patients with Acute Myocardial Infarction
}

\author{
Mihaela Susca1,2, Monica Copotoiư1,2, Horațiu Popoviciu1,2, Zsuzsanna Szőke1,3, Balázs Bajka1,4, \\ Imre Benedek ${ }^{1,3}$, Theodora Benedek ${ }^{1,3}$ \\ 1 University of Medicine and Pharmacy, Tîrgu Mureș, Romania \\ 2 Clinic of Rheumatology, County Emergency Clinical Hospital, Tîrgu Mureș, Romania \\ ${ }^{3}$ Clinic of Cardiology, County Emergency Clinical Hospital, Tîrgu Mureș, Romania \\ ${ }^{4}$ Cardio Med Medical Center, Tîrgu Mureș, Romania
}

\section{CORRESPONDENCE}

Mihaela Susca

38 Gheorghe Marinescu St

540139 Tîrgu Mureș, Romania

Tel: 0265215551

Email:mela_doc@yahoo.com

\section{ARTICLE HISTORY}

Received: 1 April, 2016

Accepted: 15 May, 2016
Monica Copotoiu - 38 Gheorghe Marinescu St, 540139 Tîrgu Mureș, Romania, Tel: +40 265215551 Horatiu Popoviciu - 38 Gheorghe Marinescu St, 540139 Tîrgu Mureș, Romania, Tel: +40 265215551 Zsuzsanna Szoke • 38 Gheorghe Marinescu St, 540139 Tîrgu Mureș, Romania, Tel: +40 265215551 Balazs Bajka • 76, 22 decembrie 1989 St, Tîrgu Mureș 540124, Romania, Tel: +40 265217333 Imre Benedek • 38 Gheorghe Marinescu St, 540139 Tîrgu Mureș, Romania, Tel: +40 265215551

Theodora Benedek • 38 Gheorghe Marinescu St, 540139 Tîrgu Mures, Romania, Tel: +40 265215551

\begin{abstract}
Background: The quality of life (QoL) in acute myocardial infarction (MI) patients can be improved using 3 therapeutic methods - surgical, pharmaceutical and physical. Study aim: We sought to assess the QoL in patients following an acute $\mathrm{Ml}$, with or without percutanous coronary intervention (PCl). Material and methods: A number of 54 patients with acute $\mathrm{Ml}$ were included in the study. All subjects were asked to complete the EQ-SD questionnaire at baseline, and during the 12-month follow-up. The questionnaire consists of 2 parts: 1st part assesses the mobility, self care, activities of daily life (ADL), pain, depression and anxiety; $2^{\text {nd }}$ part - visual analogue scale (VAS) for the overall state. Patients were divided into 4 groups: Group 1 - all patients $(n=54)$; Group 2 - males $(n=40)$, Group 3 - female patients $(n=14)$, and Group 4 - patients who underwent a PCl procedure $(n=48)$. Blood pressure $(B P)$ was also monitored. Results: The mean age was 66.54 years. There were no differences between the groups at baseline, and after 1 year regarding the BP. No differences were observed regarding the VAS (baseline $p=0.990 ; 12$-month $p=0.991$ ). Concerning the $\mathrm{PCl}$ vs. non- $\mathrm{PCl}$ groups, no differences were found in relation to mobility, self-care, ADL, pain and depression at baseline or after 12 months. For all groups at baseline, the limited mobility was positively correlated with impaired self-care $(p=0.041)$ and lower ADLs $(p=0.003)$. After 1 year, a limited mobility was associated with defective self-care $(p<0.001)$ and decreased ADLs $(p=0.004)$ and there was an improvement in mobility ( $p=0.0002)$ and self-care $(p<0.0001)$, compared to baseline. The $\mathrm{PCl}$ group associated pain with depression at baseline $(p<0.001)$ and limited mobility with lack of ADLs ( $p=0.005)$. At 12 months, we observed an improvement in mobility, self-care ( $p$ $<0.001)$, and the ADLs ( $p<0.001)$. The males showed a positive association between depression and pain $(p<0.001)$ at baseline, but not after 1 year. Mobility was the only parameter that had improved during follow-up ( $p=0.043)$. In the female group, pain $(p=0.015)$ and mobility ( $p$ $=0.033$ ) had improved after 12 months. Conclusions: The QoL had improved in terms of mobility, self-care and new skills acquired after $\mathrm{PCl}$. Both depression and pain were ameliorated in the male group, despite the lack of improvement on VAS for the overall state.
\end{abstract}

Keywords: quality of life, myocardial infarction, $\mathrm{PCl}$, 12-month follow-up 


\section{BACKGROUND}

The quality of life (QoL) in patients with at least one episode of acute myocardial infarction (MI) can be changed using rehabilitation methods (surgical, pharmaceutical and physical). Despite the international recommendations to apply all the rehabilitation strategies, less than a quater of MI patients undergo all three methods of treatment in order to improve their QoL.

The main aim of our study was to investigate the quality of life in patients following an episode of acute myocardial infarction and coronary revascularization, thus building a background for local strategies to be applied in cardiac patients, in order to fullfill their need and to upgrade their outcomes.

\section{MATERIAL AND METHODS}

\section{Study design}

A survey extended for twelve months that included $54 \mathrm{pa}-$ tients diagnosed with an episode of myocardial infarction, was conducted in the Cardiology Clinic of the Clinical County Hospital of Tîrgu Mureș.

\section{Patients}

The subjects were grouped as follows: Group 1 - all the patients enrolled in the study $(\mathrm{n}=54)$, Group 2 - male patients $(n=40)$, Group 3 - female patients $(n=14)$ and Group 4 - patients who underwent coronary revascularization with primary percutaneous coronary intervention (pPCI) $(n=48)$. The present study was conducted in accordance with the Declaration of Helsinki and was approved by the local ethics committee.

\section{Parameters followed}

EQ-5D is a QoL validated questionnaire that was completed by the patients during the screening visit and at 12 months follow-up. The questionnaire consisted of two parts: the first part was assessing mobility, self-care, ac- tivities of daily life (ADL), the degree of pain, depression and anxiety; the second part consisted of a visual analogue scale (VAS) for the patient to grade its overall state.

Blood pressure (systolic, diastolic, mean arterial pressure - MAP) was also monitored. The mean arterial blood pressure was measured as two times the diastolic value plus the systolic value, divided by three. The aimed value in order to maintain the coronary perfusion was set above $60 \mathrm{mmHg}$.

\section{Statistical analysis}

The data was analyzed using Graph Pad Prism 6.0 software. The following statistical tests were applied: Spearman rank correlation, Kruskall Wallis test and paired t test.

\section{RESULTS}

The mean age of the study population was 66.54 years. The demographic data were similar in all four groups. The blood pressures (systolic, diastolic and MAP) were similar at baseline and after 12 months in all four groups (Table 1 and Table 2). The same results were observed when the VAS for the overall state was evaluated (Table 3 ).

No differences were observed between the subtypes of patients when analyzing the VAS (baseline: $p=0.9909$, at 12 months: $p=0.991$ ).

When evaluating the correlations between patients who underwent PCI vs. non-PCI, no differences were observed in relation to the degree of mobility, self-care, ADLs, pain and depression at baseline. Furthermore, we observed no significant differences between the two groups at the 12-month follow-up. However, when analyzing all groups of patients at baseline, limited mobility was positively correlated with impaired self-care $(\mathrm{p}=0.041, \mathrm{r}=0.278)$ and lower ADLs $(p=0.003, r=0.390)$. Similar results were recorded after one year with a limited mobility linked to defective self-care $(p<0.001, r=0.566)$ and decreased ADLs $(p=0.004, r=0.380)$. Furthermore, we observed that after 12 months there was an improvement of mobility ( $\mathrm{p}=$ $0.0002)$ and self-care $(p<0.0001)$, compared to baseline.

TABLE 1. Blood pressure at baseline

\begin{tabular}{lccc}
\hline Baseline & Systolic BP & Diastolic BP & Mean BP \\
\hline All patients & $134.3 \pm 13.99$ & $82.50 \pm 7.75$ & $99.63 \pm 8.98$ \\
Male patients & $133.6 \pm 13.68$ & $82.63 \pm 8.00$ & $99.55 \pm 8.97$ \\
Female patients & $136.1 \pm 15.21$ & $82.14 \pm 7.26$ & $99.86 \pm 9.33$ \\
PCl patients & $134.5 \pm 14.15$ & $82.66 \pm 7.36$ & $99.79 \pm 8.75$ \\
P (Kruskall Wallis test) & 0.9771 & 0.9822 & 0.9982 \\
\hline
\end{tabular}


TABLE 2. Blood pressure at 12 months

\begin{tabular}{lccc}
\hline Baseline & Systolic BP & Diastolic BP & Mean BP \\
\hline All patients & $135.0 \pm 14.66$ & $82.87 \pm 8.02$ & $100.3 \pm 9.43$ \\
Male patients & $134.6 \pm 14.64$ & $83.12 \pm 8.34$ & $100.3 \pm 9.64$ \\
Female patients & $136.1 \pm 15.21$ & $82.14 \pm 7.26$ & $100.1 \pm 9.17$ \\
PCl patients & $135.3 \pm 14.89$ & $83.08 \pm 7.68$ & $100.5 \pm 9.27$ \\
P (Kruskall Wallis test) & 0.9926 & 0.9563 & 0.9944 \\
\hline
\end{tabular}

However, three parameters of QoL remained unchanged at 1 year of follow-up: ADLs $(\mathrm{p}=0.204)$, pain $(\mathrm{p}=0.086)$ and depression $(\mathrm{p}=0.092)$.

When analyzing the groups by gender, we observed that the male population showed a positive association between depression and pain ( $\mathrm{p}<0.001, \mathrm{r}=0.587)$ at baseline, but not during the 12-month follow-up. Moreover, mobility was linked with ADLs at baseline $(\mathrm{p}=0.001, \mathrm{r}=$ 0.478 ) and after 12 months. The ADLs was also correlated with self-care at 12 months. Mobility was the only parameter that had improved at follow-up compared to baseline in the male population $(\mathrm{p}=0.043)$.

The number of women included in the study was insufficient; therefore correlation analysis at baseline and 12 months could not be performed. Nevertheless, pain ( $\mathrm{p}=$ $0.015)$ and mobility ( $\mathrm{p}=0.033)$ improved after 12 months.

At screening, the PCI patients associated pain with depression $(\mathrm{p}<0.001, \mathrm{r}=0.612)$ and limited mobility with lack of ADLs ( $p=0.005, r=0.397)$. At 12 months followup, we observed an improvement in mobility and self-care ( $\mathrm{p}<0.001, \mathrm{r}=0.699)$, and also in the ADLs $(\mathrm{p}<0.001, \mathrm{r}=$ $0.699)$.

\section{DISCUSSIONS}

Starting from the results of the Gupta group that emphasized on the poorer quality of life, in terms of depression, perception of social support and health, in the case of patients with MI vs. healthy subjects, our study aimed to analyze the standpoint of MI patients only. ${ }^{1}$ As expected, we observed an impaired status of health from the screening that improved in terms of mobility and increased abilities for self-care and ADLs in both PCI and non-PCI patients. Unfortunately, the depression and pain resolution was seen only in men, with no discrimination between PCI vs. non- PCI groups. These results are in concordance with a study published by Rancic et al., who evaluated 160 patients with acute MI after 1 month of management, showing improvement in regards to pain, depression and self-care. $^{2}$

Symptoms such as physical limitation due to pain or depression due to chronic pain were improved after one year despite the evaluation of global status on the VAS scale. Those data are in concordance with the data from a study carried out by Wadie et al. ${ }^{3}$

The means of treatment such as coronary artery bypass graft (CABG) versus percutaneous coronary intervention (PCI) showed small differences in terms of QoL outcome as noticed in the FREEDOM study, therefore we were not concerned about the initial management of patients enrolled in our study (only PCI patients). Similar reports were published by Wadie et al.3,4

The main limitation of our study was the low number of non-PCI and female patients.

\section{CONCLUSIONS}

The quality of life (QoL) had improved in terms of mobility, self-care and new skills were acquired after coronary revascularization. Both depression and pain were amelio-

TABLE 3. VAS for overall state - at baseline and at 12-month follow-up

\begin{tabular}{lcc}
\hline Baseline & Baseline & 12 months \\
\hline All patients & $76.41 \pm 12.04$ & $82.26 \pm 7.49$ \\
Male patients & $76.15 \pm 12.39$ & $82.43 \pm 6.68$ \\
Female patients & $77.14 \pm 11.39$ & $81.79 \pm 9.72$ \\
PCl patients & $76.94 \pm 11.83$ & $82.77 \pm 6.90$ \\
P (Kruskall Wallis test) & 0.990 & 0.991 \\
\hline
\end{tabular}


rated in the male group, despite the lack of improvement on the VAS for overall state. In order to rule out the lowest point of the study, further enrollment of patients is to be considered (especially women and non coronary revascularization patients).

\section{CONFLICT OF INTEREST}

None declared.

\section{REFERENCES}

1. Gupta S, Das S, Sahewalla R, et al. A study on quality of life in patients following myocardial infarction. Indian J Physiol Pharmacol. 2012;56(1):28-35.

2. Rančić NK, Petrović BD, Apostolović SR, Kocić BN, Ilić MV. Health-related quality of life in patients after the acute myocardial infarction. Centr Eur $J$ Med. 2013;8(2):266-272

3. Wadie B, Shaheen S, Nashat E. Quality of life assessment after coronary artery revascularization using coronary revascularization outcome questionnaire in ischemic Egyptian patients. Med J Cairo Univ. 2013;81(1):1-5.

4. Abdallah MS, Wang K, Magnuson EA, et al. Quality of life after PCI vs CABG among patients with diabetes and multivessel coronary artery disease a randomized clinical trial. JAMA. 2013;310(15):1581-1590. 